Check for updates

Cite this: Nat. Prod. Rep., 2019, 36, 248

\title{
Correction: The value of universally available raw NMR data for transparency, reproducibility, and integrity in natural product research
}

\author{
James B. McAlpine, (DD *a Shao-Nong Chen, (iD ${ }^{a}$ Andrei Kutateladze, (D) ${ }^{b}$ \\ John B. MacMillan, (D) c Giovanni Appendino, (D) A Andersson Barison, (D) \\ Mehdi A. Beniddir, (D) ${ }^{f}$ Maique W. Biavatti, (D) ${ }^{g}$ Stefan Bluml, (D) ${ }^{\mathrm{h}}$ Asmaa Boufridi, (D) \\ Mark S. Butler, (D) ${ }^{j}$ Robert J. Capon, (D) ${ }^{j}$ Young H. Choi, (D) ${ }^{k}$ David Coppage, ${ }^{c}$ \\ Phillip Crews, (D) ${ }^{c}$ Michael T. Crimmins, (D) 'Marie Csete, (D) ${ }^{m}$ Pradeep Dewapriya, (DD ${ }^{j}$ \\ Joseph M. Egan, (DD nary J. Garson, (D) ${ }^{n}$ Grégory Genta-Jouve, (D) P \\ William H. Gerwick, (D) ar Harald Gross, (D) s Mary Kay Harper, ${ }^{t}$ Precilia Hermanto, ${ }^{u}$ \\ James M. Hook, (D) " Luke Hunter, (D) " Damien Jeannerat, (D) ${ }^{v}$ Nai-Yun Ji, (D) w \\ Tyler A. Johnson, ${ }^{c}$ David G. I. Kingston, (DD ${ }^{\times}$Hiroyuki Koshino, (D) y Hsiau-Wei Lee, ${ }^{c}$ \\ Guy Lewin, ${ }^{f} \mathrm{Jie}$ Li, (D) ${ }^{r}$ Roger G. Linington, (D) ${ }^{\mathrm{n}}$ Miaomiao Liu, ${ }^{i}$ Kerry L. McPhail, (D) ${ }^{z}$ \\ Tadeusz F. Molinski, (D) aa Bradley S. Moore, (D) ar Joo-Won Nam, (D) ab \\ Ram P. Neupane, ${ }^{\text {ac }}$ Matthias Niemitz, (D) ad Jean-Marc Nuzillard, (D) ae \\ Nicholas H. Oberlies, (D) af Fernanda M. M. Ocampos, (D) e Guohui Pan, (iD) as \\ Ronald J. Quinn, (D) i D. Sai Reddy, ' Jean-Hugues Renault, (D) ae José Rivera-Chávez, ${ }^{\text {ah }}$ \\ Wolfgang Robien, (D) ai Carla M. Saunders, (D) aj Thomas J. Schmidt, (D) ak \\ Christoph Seger, (D) al Ben Shen, (ID ag Christoph Steinbeck, (D) am \\ Hermann Stuppner, (D) al Sonja Sturm, al Orazio Taglialatela-Scafati, (D) an \\ Dean J. Tantillo, (D) aj Robert Verpoorte, (D) ${ }^{k}$ Bin-Gui Wang, (D) wao \\ Craig M. Williams, (D) Philip G. Williams, (D) ac Julien Wist, (D) ap Jian-Min Yue, (D) aq \\ Chen Zhang, ${ }^{\text {ar }}$ Zhengren Xu, (D) ag Charlotte Simmler, (D) a David C. Lankin, (D) ${ }^{\text {a }}$ \\ Jonathan Bisson (iD) a and Guido F. Pauli (iD)*a
}

DOI: $10.1039 / \mathrm{c} 8 \mathrm{np} 90041 \mathrm{~h}$ Correction for 'The value of universally available raw NMR data for transparency, reproducibility, and integrity in natural product research' by James B. McAlpine et al., Nat. Prod. Rep., 2018, DOI: 10.1039/ c7np00064b.

www.rsc.org/npr

The authors regret that there were errors within references 83-85 within the version of the article published on 13 th July 2018 . These errors have now been corrected and the corrected references are shown below:

83 J. Bredt, J. Houben and P. Levy, Ber. Dtsch. Chem. Ges., 1902, 35, 1286-1292.

84 J. Bredt, J. Houben, P. Levy and S. Link, Justus Liebigs Ann. Chem., 1913, 395, 26-63.

85 J. Bredt, H. Thouet and J. Schmitz, Justus Liebigs Ann. Chem., 1924, 437, 1-13.

The Royal Society of Chemistry apologises for these errors and any consequent inconvenience to authors and readers.

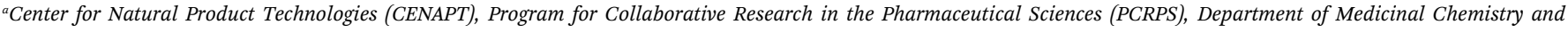
Pharmacognosy, College of Pharmacy, University of Illinois at Chicago, 833 S. Wood St., Chicago, IL, 60612, USA. E-mail: gfp@uic.edu; mcalpine@uic.edu

${ }^{b}$ Department of Chemistry and Biochemistry, University of Denver, Denver, CO, 80210, USA

${ }^{\circ}$ Department of Chemistry and Biochemistry, University of California, Santa Cruz, CA, 95064, USA

${ }^{d}$ Dipartimento di Scienze Chimiche, Alimentari, Farmaceutiche e Farmacologiche, Università del Piemonte Orientale, Via Bovio 6, 28100 Novara, Italy

${ }^{e}$ NMR Center, Federal University of Paraná, Curitiba, Brazil

${ }^{f}$ Équipe "Pharmacognosie-Chimie des Substances Naturelles” BioCIS, Univ. Paris-Sud, CNRS, Université Paris-Saclay, 5 rue J.-B. Clément, 9229o Châtenay-Malabry, France

${ }^{g}$ Department of Pharmaceutical Sciences, Federal University of Santa Catarina, Florianópolis, Brazil

${ }^{h}$ University of Southern California, Keck School of Medicine, Los Angeles, CA, 90089, USA

${ }^{i}$ Griffith Institute for Drug Discovery, Griffith University, Brisbane, QLD, 4111, Australia

${ }^{j}$ Institute for Molecular Bioscience, The University of Queensland, St. Lucia, QLD, 4072, Australia

${ }^{k}$ Division of Pharmacognosy, Section Metabolomics, Institute of Biology, Leiden University, P.O. Box 9502, 2300 RA Leiden, The Netherlands
} 
${ }^{\prime}$ Kenan and Caudill Laboratories of Chemistry, University of North Carolina at Chapel Hill, Chapel Hill, NC, 27599, USA

${ }^{m}$ University of Southern California, Huntington Medical Research Institutes, 99 N. El Molino Ave., Pasadena, CA, 91101, USA

${ }^{n}$ Department of Chemistry, Simon Fraser University, Burnaby, BC, V5A 1S6, Canada

${ }^{\circ}$ School of Chemistry and Molecular Sciences, University of Queensland, St. Lucia, QLD 4072, Australia

${ }^{p}$ C-TAC, UMR 8638 CNRS, Faculté de Pharmacie de Paris, Paris-Descartes University, Sorbonne, Paris Cité, 4, Aveue de l'Observatoire, 75006 Paris, France

${ }^{9}$ Skaggs School of Pharmacy and Pharmaceutical Sciences, University of California, La Jolla, San Diego, CA, 92093, USA

${ }^{r}$ Center for Marine Biotechnology and Biomedicine, Scripps Institution of Oceanography, La Jolla, CA, 92093, USA

sPharmaceutical Institute, Department of Pharmaceutical Biology, Eberhard Karls University of Tübingen, Auf der Morgenstelle 8, 72076 Tübingen, Germany

${ }^{t}$ Department of Medicinal Chemistry, University of Utah, Salt Lake City, UT, 84112, USA

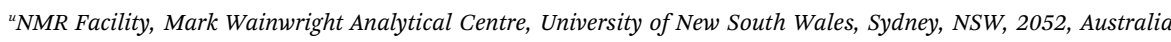

${ }^{\nu}$ University of Geneva, Department of Organic Chemistry, 30 quai E. Ansermet, CH 1211 Geneva 4, Switzerland

"Yantai Institute of Coastal Zone Research, Chinese Academy of Sciences, Chunhui Road 17, Yantai 264003, People's Republic of China

${ }^{x}$ Department of Chemistry, M/C 0212, Virginia Polytechnic Institute and State University, Blacksburg, VA, 24061, USA

${ }^{y}$ RIKEN Center for Sustainable Resource Science, Wako, Saitama, 351-0198, Japan

${ }^{2}$ Department of Pharmaceutical Sciences, College of Pharmacy, Oregon State University, Corvallis, OR, 97331, USA

${ }^{a a}$ Department of Chemistry and Biochemistry and Skaggs School of Pharmacy and Pharmaceutical Sciences, University of California, 9500 Gilman Drive MC-0358, La Jolla, San Diego, CA, 92093, USA

${ }^{a b}$ College of Pharmacy, Yeungnam University, 280 Daehak-ro, Gyeongsan, Gyeongbuk, 38541, Republic of Korea

${ }^{a c}$ Department of Chemistry, University of Hawaii at Manoa, 2545 McCarthy Mall, Honolulu, HI 96822, USA

${ }^{a d}$ NMR Solutions Limited, Puijonkatu 24B5, 70110, Kuopio, Finland

${ }^{a e}$ FRE CNRS 2715, IFR 53, Université de Reims Champagne-Ardenne, Bât. 18, Moulin de la Housse, BP 1039, 51687 Reims, Cedex 2, France

afDepartment of Chemistry and Biochemistry, University of North Carolina at Greensboro, Greensboro, NC, 27402, USA

${ }^{a g}$ Department of Chemistry, Department of Molecular Medicine, Natural Products Library Initiative at the Scripps Research Institute, Jupiter, FL 33458, USA

ah Instituto de Quimica, Universidad Nacional Autónoma de México, Ciudad de México 04510, Mexico

${ }^{a i}$ University of Vienna, Department of Organic Chemistry, Währingerstrasse 38, A-1090 Vienna, Austria

${ }^{a j}$ Department of Chemistry, University of California, Davis, One Shields Avenue, Davis, CA, 95616, USA

${ }^{a k}$ Institute of Pharmaceutical Biology and Phytochemistry (IPBP), University of Münster, Pharma Campus, Corrensstrasse 48, D-48149 Münster, Germany

${ }^{a l}$ Institute of Pharmacy, Pharmacognosy, Member of CMBI, University of Innsbruck, Innrain 80-82, 6020 Innsbruck, Austria

${ }^{a m}$ Institute of Inorganic and Analytical Chemistry, Friedrich-Schiller-University, D-07743 Jena, Germany

${ }^{a n}$ Dipartimento di Farmacia, Università; di Napoli Federico II, Via Montesano 49, 80131 Napoli, Italy

${ }^{a}$ Laboratory of Marine Biology and Biotechnology, Qingdao National Laboratory for Marine Science and Technology, Key Laboratory of Experimental Marine Biology, Institute of Oceanology, Chinese Academy of Sciences, Nanhai Road 7, Qingdao 266071, People's Republic of China

${ }^{a p}$ Departamento de Química, Universidad del Valle, AA 25360, Cali, Colombia

${ }^{a q}$ State Key Laboratory of Drug Research, Shanghai Institute of Materia Medica, Chinese Academy of Sciences, 555 Zu Chong Zhi Road, Zhangjiang Hi-Tech Park, Shanghai 201203, People's Republic of China

arDepartment of Nanoengineering, University of California, La Jolla, San Diego, CA, 92093, USA 